\title{
The analysis of echocardiographic results in patients suffering from epidermolysis bullosa
}

\author{
Katarzyna Kurnickaํ, Katarzyna Osipowicz², Olga Dzikowska-Diduch¹, Katarzyna Wertheim-Tysarowska ${ }^{3}$, \\ Cezary Kowalewski², Piotr Pruszczyk ${ }^{1}$
}

'Department of Internal Medicine and Cardiology, Medical University of Warsaw, Warsaw, Poland 2Department of Dermatology and Immunodermatology, Medical University of Warsaw, Warsaw, Poland ${ }^{3}$ Department of Medical Genetics, Institute of Mother and Child, Warsaw, Poland

Adv Dermatol Allergol 2020; XXXVII (6): 871-878 DOI: https://doi.org/10.5114/ada.2020.102101

\begin{abstract}
Introduction: Cardiac abnormalities revealed in patients suffering from epidermolysis bullosa (EB) include dilated cardiomyopathy (DC) and aortopathy. DC is a rare but serious complication associated with an increased mortality, predominantly observed in recessive dystrophic EB. Echocardiography is the most available diagnostic tool used to detect heart disease in EB patients.

Aim: To analyse echocardiographic results obtained in Polish EB patients and compare them between the EB group and healthy persons.

Material and methods: We analysed retrospectively echocardiograms of 23 patients with EB (14 F, mean age 17.3 years) performed from 2017 to 2019. The incidence of left ventricular (LV) systolic and diastolic dysfunction, right heart disease and congenital heart disease was evaluated. A comparison of echo-parameters between EB patients and 20 matched healthy subjects was performed.

Results: We did not find any cases of DC and aortopathy in the EB group. One bicuspid aortic valve case was revealed. Analysis of $\mathrm{LV}$ diastolic parameters showed that the mean value of mitral A velocity was significantly higher and the pulmonary venous flow D velocity was lower in the EB group than in controls. Tissue Doppler analysis revealed lower values of E' velocities of mitral annulus in the EB group, what may suggest discreetly slower LV relaxation, however, this will definitely require further research.

Conclusions: Although most EB patients do not present cardiac symptoms, there is still a risk of developing cardiomyopathy associated with poor prognosis. It seems reasonable to perform a scheduled echocardiographic screening including LV systolic and diastolic function assessment to detect preclinical cardiac abnormalities.
\end{abstract}

Key words: epidermolysis bullosa, echocardiography, cardiomyopathy.

\section{Introduction}

Epidermolysis bullosa (EB) is a rare inherited disease characterized by recurrent blister formation as a result of structural fragility within the skin [1]. Its clinical manifestations may include blisters on the skin of the hands and feet, in the oral cavity and oesophagus [1, 2].

There are four major types of EB: simplex (EBS), junctional (JEB), dystrophic (DEB) and Kindler syndrome and at least 30 subtypes [3].
Types of EB are defined based on mutations within the genes encoding for several different proteins, involved in the adhesion and structural stability of keratinocytes $[1,2]$. Specific mutated proteins such as keratins 5 and 14 (EBS), integrin $\alpha 6 \beta 4$, collagen XVII (JEB) and collagen VII (DEB) are present in certain types [3-5].

In recent years, quite a few papers have been published regarding cardiac abnormalities in EB patients including cardiomyopathies, aortopathy and arrhythmias [6-9]. Cases of dilated cardiomyopathy (DC), defined as a significant left ventricle (LV) systolic dysfunction and its enlargement, were

Address for correspondence: Assoc. Prof. Katarzyna Kurnicka, Department of Internal Medicine and Cardiology, Medical University of Warsaw, Warsaw, Poland, phone: +48 2250217 96, e-mail: kkurnicka@yahoo.pl

Received: 28.05.2020, accepted: 12.08.2020. 
most frequently reported [10-12]. This serious complication was predominantly observed in recessive DEB (RDEB) and also in JEB and EBS [12-15]. Transthoracic echocardiography (TTE) was usually a diagnostic tool used to detect heart disease. Although DC is a rare complication in EB patients, evidence has been provided that it is associated with an increased mortality $[8,10,16,17]$.

\section{Aim}

The goal of study was to analyse echocardiographic results obtained in Polish patients suffering from EB to detect cardiac abnormalities and to compare them between EB patients and matched healthy persons.

To our best knowledge, this is the first echocardiographic analysis aimed to evaluate cardiac function in a unique group of EB patients performed in Poland.

\section{Material and methods}

We analysed retrospectively transthoracic echocardiograms of 23 patients suffering from epidermolysis bullosa (14 females (60.9\%), mean age: $17.3 \pm 10.2$ years) referred by a dermatologist to the cardiology department, which were performed from May 2017 to July 2019. The patients were genotyped in the Department of Medical Genetics of the Institute of Mother and Child using Sanger sequencing or next generation sequencing (NGS) with EB gene panel. The mutations were named according to HGVS nomenclature. The pathogenicity status of novel variants was assessed using ACMG classification available at Varsome website https://varsome.com/. For most patients it was the first TTE examination.

Primarily, we aimed to evaluate the incidence of the left ventricular systolic and diastolic dysfunction, right heart disease and congenital heart disease in the EB group. Then, we made a comparison of echocardiographic parameters found in EB patients with those obtained in 20 healthy subjects matched by gender and age (11 females (61.1\%), mean age: $17.0 \pm 7.7$ years), which were previously examined in our centre. In all participants, a clinical examination and a measurement of heart rate $(\mathrm{HR})$, body mass index (BMI) and body surface area (BSA) were performed immediately before the TTE. Blood pressure (BP) was measured in 18 patients with $\mathrm{EB}(78 \%)$ and in the remaining participants it was not taken due to the high risk of skin damage. Additionally, a rest 12-lead ECG recordings of 17 patients with EB (74\%) were assessed.

This analysis was approved by the Ethics Committee of the Medical University of Warsaw.

\section{Echocardiography}

Echocardiography (TTE) images were obtained using the EPIQ 7 system (Phillips, Eindhoven, NL). The quality of TTE recordings was good and adequate for the analysis in all participants. The findings were interpreted by an experienced physician according to the recommendations of the Echocardiography Working Group of the Polish Cardiac Society and to the guidelines of the European Association of Cardiovascular Imaging $[18,19]$.

The left ventricle morphology and systolic function were assessed. LV ejection fraction (EF) was calculated using the modified Simpson's rule [19]. Additionally, mitral annulus peak systolic excursion (MAPSE) and mitral annulus maximal systolic velocity (MV S') by tissue Doppler imaging (TDI) were measured, to evaluate longitudinal LV function. Colour Doppler images were analysed in order to assess the function of the valves and to spot the presence of congenital cardiac shunts.

LV diastolic function was evaluated using Doppler mitral valve flow (MVF) parameters (maximal velocity of the early (E) and the atrial wave (A), E/A ratio, E-wave deceleration time (DCT)) and pulmonary venous flow (PVF) parameters (maximal systolic (S), diastolic (D) and atrial reversal (Arev) velocities) and left atrial (LA) dimension.

Early diastolic velocities of the lateral (E' lat) and septal (E' sept) parts of the mitral annulus were measured and average $E / E$ ' ratios were derived. LV diastolic dysfunction was defined according to the current echocardiographic recommendations [20].

LV tissue Doppler Tei index for both parts of the mitral annulus was estimated to reflect global LV function, according to the Tei formula and defined as the sum of the isovolumic contraction time and isovolumic relaxation time divided by the ejection time [21, 22].

To evaluate the right ventricle (RV) systolic function tricuspid annulus peak systolic excursion (TAPSE) and tricuspid annulus maximal systolic velocity (TV S') were measured [19]. The RV diastolic function was assessed in a similar manner like for the LV, based on tricuspid valve inflow parameters ( $E$, A, E/A, DT) and tricuspid annular velocities (E', A'). Global RV function was defined on the basis of the calculation of the Tei index in TDI. Tricuspid regurgitation peak gradient was measured using continuous wave Doppler.

Doppler measurements reflected an average of 3 cardiac cycles. A carefully selected echocardiograms of healthy persons were referential as they reflected normal values. In children (age $<18$ years), a Z-score was used in the evaluation of adequate cardiac morphology parameters [23].

\section{Statistical analysis}

Descriptive statistics for quantitative data were presented as means \pm standard deviations. Qualitative data were shown as counts and percentages. Within-group comparisons for quantitative data were performed using the repeated measures $t$-test. In between-subject comparisons the Mann-Whitney test was used. All reported results are based on two-sided statistical tests. Effects were considered significant at $p$-value less than 0.05. The statistical analyses were performed using R software (R Core Team, 2017), ver. 3.4.2. 


\section{Results}

The following types of EB were presented in analysed patients:

1) dystrophic epidermolysis bullosa (DEB) in 17 (73.9\%) patients, including a recessive type RDEB- generalized severe in 15 (65.2\%) patients and a dominant type DDEB in $2(8.7 \%)$ patients with a mutated gene of collagen VII (COL 7A1);

2) junctional EB (JEB) in 4 (17.4\%) patients with a mutation in the gene of collagen XVII (COL $17 \mathrm{~A} 1)$ in 3 patients and of laminin 332 in 1 patient;

3) Simplex EB (EBS) in $2(8.7 \%)$ patients with mutated gene of keratin 5 (KRT5). The types and genotypes of patients with EB are presented in Table 1.
No symptoms and signs of heart failure or other cardiovascular diseases were found in the EB patients. No significant differences in the HR, BP and BSA between EB patients and controls were found. However, both the weight and BMI were significantly lower in EB patients than in healthy controls. Table 2 presents basic clinical characteristics of the studied groups. There were no abnormalities in the standard rest 12-lead ECG records performed in EB patients.

\section{Echocardiography}

No cases of dilated cardiomyopathy were found in the EB group. All EB patients presented normal LV ejection fraction.

Table 1. The types and genotypes of patients with epidermolysis bullosa

\begin{tabular}{|c|c|c|c|c|c|c|}
\hline Pt & G & Age & Type EB & Gene & Allele 1 & Allele 2 \\
\hline 1 & $\mathrm{~F}$ & 5 & EBJ & COL17A1 & $\begin{array}{c}\text { c.779delC/- } \\
\text { (p.Pro260GlnfsTer32) }\end{array}$ & Unknown \\
\hline 2 & $\mathrm{~F}$ & 9.5 & EBJ & COL17A1 & $\begin{array}{c}\text { c.1826G>A } \\
\text { (p.Gly609Asp) }\end{array}$ & $\begin{array}{l}\text { c.1490_1491delinsT } \\
\text { p.Ala497ValfsTer23 }\end{array}$ \\
\hline 3 & $\mathrm{~F}$ & 33 & EBJ & COL17A1 & $\begin{array}{c}\text { c.3487G >T } \\
\text { (p.Glu1163Ter) }\end{array}$ & c.1490_1491delinsT (p.Ala497ValfsTer23) \\
\hline 4 & M & 36 & EBJ & $L A M B 3$ & $\begin{array}{l}\text { c.1439_1443delCGTGT } \\
\text { (p.Pro480ArgfsTer54) }\end{array}$ & $\begin{array}{l}\text { c.1439_1443delCGTGT } \\
\text { (p.Pro480ArgfsTer54) }\end{array}$ \\
\hline 5 & $\mathrm{~F}$ & 11 & RDEB-HS & COL7A1 & c. $425 A>G$ (p.Lys142Arg & c.8038G>A (p.Gly2680Ser) \\
\hline 6 & M & 21.5 & RDEB-HS & COL7A1 & c.425A>G (p.Lys142Arg) & c.425A>G (p.Lys142Arg) \\
\hline 7 & M & 15.5 & RDEB-HS & COL7A1 & c.425A>G (p.Lys142Arg) & c. $682+1 G>A$ \\
\hline 8 & $\mathrm{~F}$ & 17.5 & RDEB-HS & COL7A1 & c.425A>G (p.Lys142Arg) & c.425A>G (p.Lys142Arg) \\
\hline 9 & M & 13 & RDEB-HS & COL7A1 & c.425A>G (p.Lys142Arg) & c.425A>G (p.Lys142Arg) \\
\hline 10 & $\mathrm{~F}$ & 21 & RDEB & COL7A1 & c.425A>G (p.Lys142Arg) & $\begin{array}{c}\text { c.5086C }>T+\text { c.5114C }>T \\
\text { (p.Arg1696Cys+) p.Pro1705Leu) }\end{array}$ \\
\hline 11 & M & 26.5 & RDEB & COL7A1 & c.425A>G (p.Lys142Arg) & $\begin{array}{c}\text { c.5086C }>T+\text { c.5114C }>T \\
\text { (p.Arg1696Cys+) p.Pro1705Leu) }\end{array}$ \\
\hline 12 & $\mathrm{~F}$ & 41.5 & DDEB & COL7A1 & c.6127G>A (p.Gly2043Arg) & wt \\
\hline 13 & $\mathrm{~F}$ & 11.5 & RDEB & COL7A1 & c. $5154+1 G>T$ & c. $5154+1 G>T$ \\
\hline 14 & M & 5.5 & RDEB & COL7A1 & c.425A>G (p.Lys142Arg) & c.7154delC (p.Pro2385ArgfsTer36) \\
\hline 15 & M & 16.5 & RDEB & COL7A1 & c.425A>G (p.Lys142Arg) & c.425A>G (p.Lys142Arg) \\
\hline 16 & $\mathrm{~F}$ & 19 & RDEB & COL7A1 & c. $682+1 G>A$ & c. $5532+6 \mathrm{~T}>\mathrm{C}^{*}$ \\
\hline 17 & $\mathrm{~F}$ & 16 & RDEB & COL7A1 & c. $682+1 G>A$ & c. $5532+6 \mathrm{~T}>\mathrm{C}^{*}$ \\
\hline 18 & $\mathrm{~F}$ & 18 & EBS WC & KRT5 & c.508G>A (p.Glu170Lys) & c.508G>A (p.Glu170Lys) \\
\hline 19 & M & 8.5 & EBS & KRT5 & c.1410C>G (p.Tyr470Ter) & wt \\
\hline 20 & $\mathrm{~F}$ & 6 & DDEB & COL7A1 & c.6127G>A (p.Gly2043Arg) & wt \\
\hline 21 & $\mathrm{~F}$ & 27.5 & RDEB & COL7A1 & c.425A>G (p.Lys142Arg) & c.5884A>C* (p.Thr1962Pro) \\
\hline 22 & $\mathrm{~F}$ & 9 & RDEB & COL7A1 & c.2387G>A p.Trp796Ter & c.2387G >A p.Trp796Ter \\
\hline 23 & M & 6.5 & RDEB & COL7A1 & c.2387G>A p.Trp796Ter & c.2387G >A p.Trp796Ter \\
\hline
\end{tabular}


Table 2. Basic clinical parameters of EB patients and healthy controls

\begin{tabular}{lccc}
\hline Parameter & EB $(n=23)$ & Controls $(n=20)$ & $P$-value \\
\hline Weight $[\mathrm{kg}]$ & $36.4 \pm 15.7$ & $46.2 \pm 15.7$ & 0.05 \\
\hline Height $[\mathrm{cm}]$ & $149.8 \pm 23.2$ & $155.2 \pm 18.0$ & 0.4 \\
\hline BMI $\left[\mathrm{kg} / \mathrm{m}^{2}\right]$ & $15.4 \pm 3.2$ & $18.5 \pm 2.9$ & 0.003 \\
\hline BSA $\left[\mathrm{m}^{2}\right]$ & $1.21 \pm 0.35$ & $1.4 \pm 0.32$ & 0.085 \\
\hline HR $[$ beats $/ \mathrm{min}]$ & $78.4 \pm 7.0$ & $77.6 \pm 8.4$ & 0.75 \\
\hline Parameter & EB $(n=18)$ & Controls $(n=20)$ & $P$-value \\
\hline BP syst $[\mathrm{mm} \mathrm{Hg}]$ & $108.6 \pm 13.1$ & $110.2 \pm 13.9$ & 0.7 \\
\hline BP diast $[\mathrm{mm} \mathrm{Hg}]$ & $69.7 \pm 7.4$ & $69.7 \pm 8.5$ & 0.98
\end{tabular}

$B M I$ - body mass index, BSA - body surface area, $H R$ - heart rate, $B P$ - blood pressure.

Bicuspid aortic valve was detected in 1 patient with EB (4.3\%). No other congenital heart diseases or pathology of the aorta were found (Figure $1 \mathrm{~A}$ ).

Most of the subjects presented small physiological mitral and tricuspid regurgitation (Figure 1 B). Mean values of basic cardiac chambers and aortic dimensions did not differ significantly between EB patients and healthy controls. However, mean values of the interventricular septum and posterior wall thickness were lower in the EB group but without statistical significance (Table 3 ).

Z-score evaluation revealed in 9 of 13 EB patients aged $<18$ years (69.2\%) a lower LV end diastolic diameter
(LVEDD) than expected. Such a result was confirmed only in 3 of 12 controls < 18 years (25\%).

We did not reveal any differences in mean values of LV systolic function parameters such as EF, S' velocities of both parts of the mitral annulus and MAPSE between groups (Table 4).

A detailed analysis of LV diastolic parameters showed that the mean value of MVF A velocity was significantly higher and the PVF D velocity was significantly lower in the EB group than in controls. In TDI assessment the mean values of septal MV E' velocity were significantly lower and septal E/E' ratio was significantly higher in EB patients.

The mean values of lateral MV E' velocity (Figure 1 C) were also lower in the EB group, however, on the border of statistical significance. Thus, the mean value of average E/E' was significantly higher in the EB group. There were no cases of LV diastolic dysfunction defined according to current recommendations. The left ventricular Tei index (TDI) was similar in both groups (Table 4).

The comparison of RV systolic function parameters including TAPSE (Figure 1 D) and RV S' showed no differences. In the RV diastolic function analysis, mean values of tricuspid valve $A$ and $A^{\prime}$ velocities were significantly higher than in controls, probably due to RV hyperkinesis. The right ventricular Tei index (TDI) was similar in the compared groups. Cases with elevated calculated right ventricular systolic pressure were not found (Table 5).
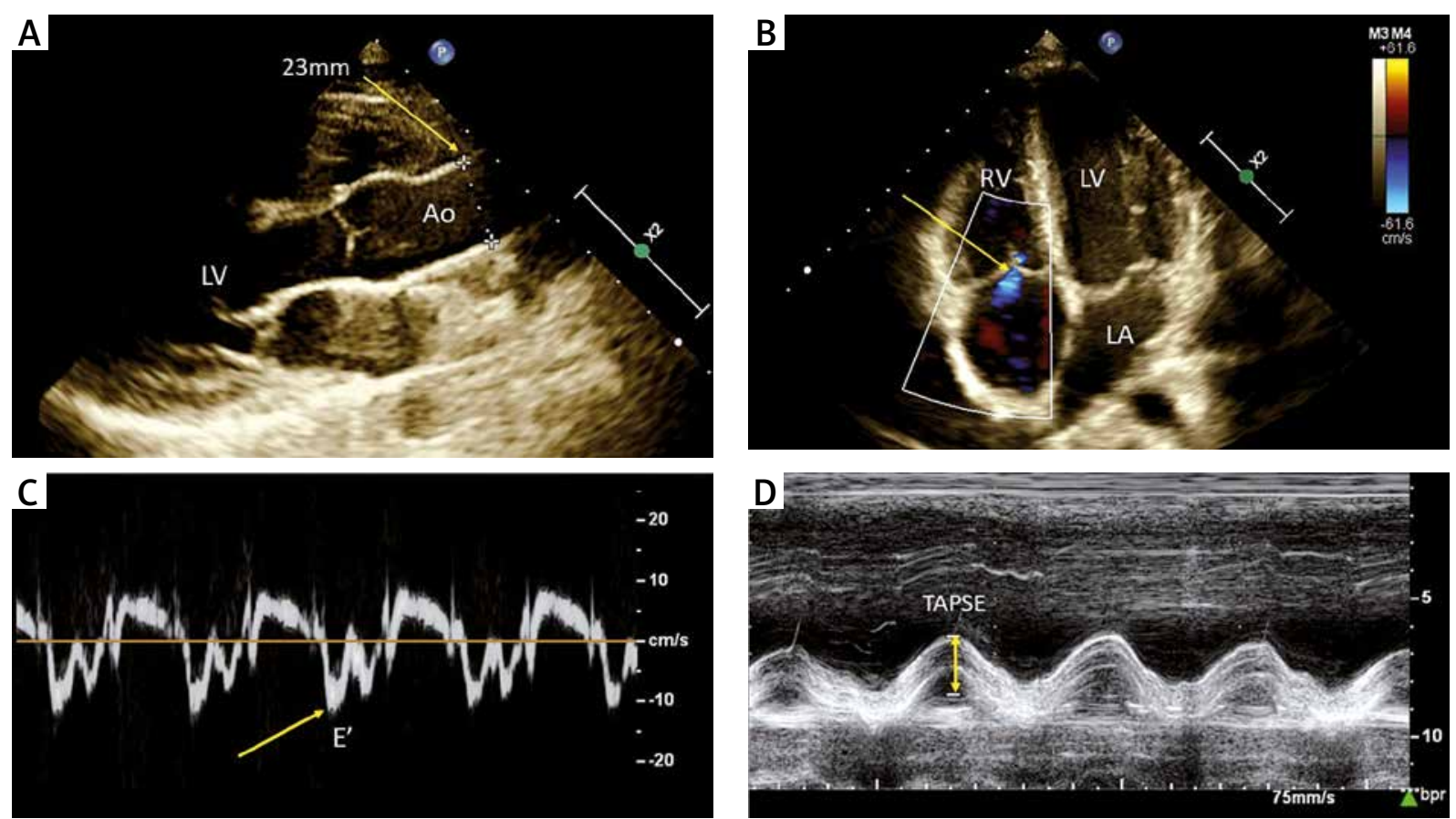

Figure 1. Echocardiographic measurements in patients with epidermolysis bullosa. A - Correct diameter of the ascending aorta (arrow), B - colour Doppler: physiological tricuspid regurgitation, C - tissue Doppler Imaging: maximal early diastolic velocity of the lateral mitral annulus - E' (arrow), D - M-mode presentation: tricuspid annulus peak systolic excursion - TAPSE (arrow) 
Table 3. Echocardiographic dimensions in EB patients and healthy controls

\begin{tabular}{lccc}
\hline Parameter & EB $(n=23)$ & Controls $(n=20)$ & $P$-value \\
\hline Left ventricle: & & & \\
\hline LA a-p diameter [mm] & $23.3 \pm 3.7$ & $24.1 \pm 3.6$ & 0.54 \\
\hline LVEDD [mm] & $37.3 \pm 6.3$ & $38.9 \pm 4.4$ & 0.35 \\
\hline IVS [mm] & $6.9 \pm 1.3$ & $7.7 \pm 1.4$ & 0.07 \\
\hline PW [mm] & $6.8 \pm 1.2$ & $7.4 \pm 1.1$ & 0.08 \\
\hline Aortic bulb [mm] & $24.4 \pm 5.8$ & $24.8 \pm 3.7$ & 0.8 \\
\hline Ascending aorta [mm] & $20.8 \pm 4.2$ & $22.7 \pm 3.2$ & 0.14 \\
\hline Aortic arch [mm] & $19.6 \pm 3.0$ & $19.9 \pm 3.0$ & 0.71 \\
\hline Right ventricle: & & & \\
\hline RVOT proximal [mm] & $21.9 \pm 4.0$ & $21.8 \pm 5.3$ & 0.98 \\
\hline
\end{tabular}

IVS - interventricular septum, LA - left atrium, LVEDD - left ventricular diastolic diameter, PW - posterior wall, RVOT - right ventricular outflow tract.

Table 5. Right ventricular function parameters in EB patients and healthy controls

\begin{tabular}{lccc}
\hline Parameter & EB $(n=23)$ & Controls $(n=20)$ & $P$-value \\
\hline $\begin{array}{l}\text { Systolic function: } \\
\text { TAPSE }[\mathrm{mm}]\end{array}$ & $22.2 \pm 2.9$ & $23.5 \pm 3.2$ & 0.2 \\
\hline TV S' vel [cm/s] & $14.1 \pm 1.71$ & $13.4 \pm 1.3$ & 0.2 \\
\hline Diastolic function: & & & \\
\hline TV E vel [cm/s] & $68.0 \pm 14.0$ & $61.1 \pm 8.1$ & 0.07 \\
\hline TV A vel [cm/s] & $44.4 \pm 11.0$ & $34.2 \pm 4.2$ & 0.001 \\
\hline TV E/A ratio & $1.62 \pm 0.53$ & $1.8 \pm 0.3$ & 0.17 \\
\hline TV E' $[\mathrm{cm} / \mathrm{s}]$ & $16.3 \pm 3.5$ & $15.5 \pm 2.9$ & 0.5 \\
\hline TV A' $[\mathrm{cm} / \mathrm{s}]$ & $11.2 \pm 2.7$ & $9.0 \pm 1.9$ & 0.005 \\
\hline TV E/E' & $3.7 \pm 1.6$ & $3.9 \pm 0.6$ & 0.48 \\
\hline TV Tei Index & $0.50 \pm 0.11$ & $0.48 \pm 0.1$ & 0.6 \\
\hline TRPG [mm Hg] & $14.9 \pm 4.3$ & $15.7 \pm 3.5$ & 0.59
\end{tabular}

A-maximal velocity of the atrial wave, $A^{\prime}$ - tricuspid annular late diastolic velocity, $E$ - maximal velocity of the early wave, E'-tricuspid annular early diastolic velocity, S' - tricuspid annulus maximal systolic velocity, TAPSE - tricuspid annulus peak systolic excursion, TRPG - tricuspid regurgitation peak gradient, TV - tricuspid valve.

We found no significant differences between the DEB subgroup (15 patients, 65.2\%) and remaining EB patients.

\section{Discussion}

Extracutaneous involvement in epidermolysis bullosa may include not only muscular dystrophy, lung fibrosis, nephrotic syndrome but also cardiomyopathy [4]

Transthoracic echocardiography is the basic and widely available diagnostic method of heart diseases useful in EB patients. Abnormal echocardiographic findings such as LV hypertrophy, LV dilated cardiomyopathy,
Table 4. Left ventricular function parameters in EB patients and healthy controls

\begin{tabular}{|c|c|c|c|}
\hline Parameter & $\mathrm{EB}(n=23)$ & Controls $(n=20)$ & $P$-value \\
\hline \multicolumn{4}{|l|}{ Systolic function: } \\
\hline LV EF (\%) & $68.6 \pm 1.5$ & $69.1 \pm 1.4$ & 0.29 \\
\hline MV S' lat vel [cm/s] & $12.3 \pm 2.2$ & $12.2 \pm 1.96$ & 0.91 \\
\hline MV S' sept vel [cm/s] & $9.0 \pm 1.3$ & $9.1 \pm 1.3$ & 0.90 \\
\hline MAPSE $[\mathrm{mm}]$ & $15.8 \pm 1.6$ & $16.2 \pm 1.6$ & 0.39 \\
\hline \multicolumn{4}{|l|}{ Diastolic function: } \\
\hline E vel $[\mathrm{cm} / \mathrm{s}]$ & $99.6 \pm 15.7$ & $96.9 \pm 9.0$ & 0.5 \\
\hline A vel $[\mathrm{cm} / \mathrm{s}]$ & $60.9 \pm 13.2$ & $51.6 \pm 7.0$ & 0.01 \\
\hline E/A ratio & $1.7 \pm 0.51$ & $1.9 \pm 0.26$ & 0.12 \\
\hline E DT [ms] & $153.7 \pm 17.3$ & $165.7 \pm 20.8$ & 0.06 \\
\hline E' lat $[\mathrm{cm} / \mathrm{s}]$ & $17.1 \pm 2.8$ & $18.7 \pm 2.3$ & 0.053 \\
\hline E/E' lat & $6.0 \pm 1.46$ & $5.3 \pm 0.93$ & 0.06 \\
\hline E' sep $[\mathrm{cm} / \mathrm{s}]$ & $12.9 \pm 2.0$ & $14.1 \pm 1.5$ & 0.045 \\
\hline E/E' sept & $7.8 \pm 1.35$ & $7.0 \pm 1.0$ & 0.025 \\
\hline Average E/E' & $6.9 \pm 1.0$ & $6.1 \pm 0.8$ & 0.02 \\
\hline $\mathrm{PV} \mathrm{S}[\mathrm{cm} / \mathrm{s}]$ & $52.0 \pm 9.3$ & $55.9 \pm 9.5$ & 0.22 \\
\hline $\mathrm{PV} D[\mathrm{~cm} / \mathrm{s}]$ & $58.2 \pm 10.6$ & $65.1 \pm 8.8$ & 0.042 \\
\hline$A \operatorname{rev}[\mathrm{cm} / \mathrm{s}]$ & $23.5 \pm 4.5$ & $22.5 \pm 3.7$ & 0.49 \\
\hline \multicolumn{4}{|l|}{ Global LV function: } \\
\hline MV Tei Index lat & $0.47 \pm 0.10$ & $0.48 \pm 0.11$ & 0.67 \\
\hline MV Tei Index sept & $0.50 \pm 0.09$ & $0.51 \pm 0.08$ & 0.92 \\
\hline
\end{tabular}

A - maximal velocity of the atrial wave, A rev-pulmonary venous atrial rever sal velocity, $D$ - pulmonary venous flow diastolic velocity, DT-deceleration time of the $E$ wave, E- maximal velocity of the early wave, E' - mitral annula early diastolic velocity, EF- ejection fraction, $L V$ - left ventricle, MAPSE - mitral annulus peak systolic excursion, MV - mitral valve, $S^{\prime}-$ mitral annulus maximal systolic velocity, $S$ - pulmonary venous flow systolic velocity.

LV non-compaction cardiomyopathy, right ventricular systolic dysfunction and aortic root dilation have been reported in RDEB and EBS patients [7, 24].

Interestingly, quite numerous case reports suggest that dilated cardiomyopathy, defined as LV enlargement and significant systolic dysfunction in the absence or evidence of other heart disease is a serious complication observed in EB patients associated with poor prognosis [25]. Of note, the incidence of DC in the paediatric population is low (0.6 to 0.7 per 10,000 children) [11].

The first report of a 5-year-old patient with DEB who died of DC complicated by LV thrombus was published by Sharratt in 1986 [26]. Then Melville et al. reported two malnourished unrelated children with autosomal RDEB who developed fatal DC [16].

Sidwell et al. observed 61 children with EB and reported a development of DCM in $6(9.8 \%)$ patients with RDEB over the period of 7 years. Three of them have died due to rapid deterioration of heart failure [27]. 
However, in the report of Batalla et al. regarding the occurrence of DC in EB patients treated in a reference hospital in Barcelona between 1986 and 2015, DC was found in only 2 RDEB (3.5\%) patients out of all 57 analysed and 23 patients who presented risk factors for potential onset of heart disease. Despite the small number of cases of DC detected, authors recommended periodic follow-up for its early diagnosis [12].

Fine et al. tried to determine the risk of heart failure or cardiomyopathy in each major EB subtype (data collection performed by the National EB Registry, from the continental US, 1986-2002) and showed that DC and HF are rare complications in both major RDEB subtypes, with the highest risk in RDEB-HS patients and also in nonHerlitz JEB, and that $30 \%$ of patients with severe RDEB and with cardiac complications died as a result of the heart disease [25].

We reviewed echocardiographic data of 23 Polish patients suffering from EB to confirm presence or absence of cardiac alterations. We have not found LV enlargement and LV systolic dysfunction in the analysed echocardiographic material, thus we have not confirmed any case of DC.

Ryan et al. found an increased LV end-diastolic diameter in $18 \%$ of EB patients and decreased LV EF in $11 \%$ of patients, however, they analysed echocardiograms in a group of patients twice as large as ours (45 RDEB patients, mean age: $12.5 \pm 7.5$ years) [24].

In our EB echocardiograms only 1 case of bicuspid aortic valve was detected (4.3\%). Dimensions of all parts of the aorta were in the normal range in contrast to the results reported by Ryan et al., who found a dilated aortic root in $18 \%$ of RDEB patients [24].

We also compared TTE results between EB patients and 20 healthy subjects matched by gender and age. We did not reveal any differences in mean values of LV ejection fraction and other parameters of LV longitudinal function such as mitral annulus S' velocities and MAPSE and LV global function such as Tei index. Analyses of similar parameters in the group of patients with EB are not available.

We have expanded our analysis to include the measurement of LV diastolic function parameters and did not detect any case of diastolic dysfunction in the EB group.

In our analysis, a comparison of TDI parameters revealed lower values of MV E' velocities of the lateral and septal part of mitral annulus in the EB group than in healthy persons, what may suggest discreetly slower LV relaxation, however, this will definitely require further research.

Data on LV diastolic function in EB patients are insufficient and it seems justified to assess this in detail.

Analysis of Ryan et al. indicated that RV dysfunction is present in some EB patients, based on decreased TAPSE found in 2 of 19 patients (11\%) [24]. No cases of RV dysfunction and elevated right ventricular systolic pressure were found in our study.
Heart disease, especially dilated cardiomyopathy in some cases leads to a fatal outcome $[8,17]$. It has been shown that DC can be diagnosed at different ages in RDEB patients (2-28 years old), sometimes in very young children, usually at the advanced stage and may result in a high mortality (30-60\%) [8, 12]. Ibernon-Moya et al. presented a 6-year-old child with RDEB admitted to ICU due to cardiomegaly, pulmonary oedema, decreased EF and cardiogenic shock with a progressive worsening of the clinical status and death despite appropriate intensive treatment [8]. Grilletta described a case of a 14-yearold girl with EBS-KLHL24 with DC and symptoms of HF, with EF 19\%, who required implantation of a LV assist and then underwent heart transplantation [15]. A 14-yearold boy with RDEB and EF $12 \%$ who did not respond to HF treatment and died within 1 month was reported by Shipman et al. [17].

On the other hand, some patients with EB and heart disease may remain asymptomatic. In Schwieger-Brel's study including 20 patients with EBS-KLHL24, 17 (85\%) patients had evidence of cardiac involvement, and $8(40 \%)$ patients had documented DC, 4 of whom had no cardiac symptoms [28].

Slow development of heart disease as well as less physical activity associated with skin problems may contribute to a lack of obvious symptoms. Unfortunately, a rapid progression and development of heart failure may occur.

Thus, it seems reasonable to perform regular noninvasive echocardiographic examinations in EB patients to identify presymptomatic cases of heart failure.

There is no established algorithm for performing cardiac evaluation in patients with EB because the majority of patients did not reveal any signs of heart disease.

Some authors advocate a routine screening for DC including TTE, electrocardiograms and biomarkers of HF such as the brain natriuretic peptide level in EB patients $[12,15,27]$.

Interestingly, Auckburally et al. tried to determine the frequency of TTE screening in patients with severe types of EB. They analysed retrospectively TTE results of $21 \mathrm{pa}-$ tients (18 with RDEB, 3 with EBS-desmoplakin deficiency) in 2006-2016 and found 4 DC cases, including 3 EBS-dd (100\%) and 1 RDEB gen-sev (5.6\%). All 4 DC patients were asymptomatic before their first TTE. The mean time between their last normal and first abnormal echocardiogram was 1.67 years. Echocardiographic abnormalities occurred at different ages of the patients, from 2 to 9.28 years old. Therefore, authors recommended cardiac screening in EBS-dd patients every 6 months from birth and annual screening in RDEB gen-sev patients [29].

Batalla et al. proposed annual cardiac evaluation in all EB patients and Oh et al. in children under 10 years of age $[10,12]$.

No cardiac symptoms were found in EB patients analysed in our study. We did not find significant heart dis- 
ease by echocardiography. However, in most patients it was the first heart examination and TTE follow-up should be continued.

It seems to be mandatory to perform TTE in all symptomatic EB patients as soon as possible, and reasonable in the rest of EB patients every 1-2 years to reveal a possible presymptomatic stage. In our opinion, more prospective studies are required to improve an algorithm of diagnostics and then appropriate treatment of patients with EB complicated by cardiac involvement.

This is a single centre observational study which included echocardiograms of a relatively small number of patients, however, it must be emphasized that EB is a very rare disease. Despite promising positive results of echocardiography in our EB patients, further cardiac investigation with a longer-term follow-up should be continued to detect preclinical cases of heart disease. Cooperation with other treatment centres for patients with EB may be also valuable.

It would be interesting to evaluate global longitudinal strain (GLS) in EB patients using speckle-tracking echocardiography, however it depends on optimal visualization in a single view and good quality of ECG monitoring, which is not always possible in patients with skin disease.

\section{Conclusions}

Although most patients with epidermolysis bullosa do not present cardiac symptoms, there is still a risk of developing cardiomyopathy associated with poor prognosis.

It seems reasonable to perform a scheduled echocardiographic screening including LV systolic and diastolic function assessment to detect preclinical cardiac abnormalities.

\section{Acknowledgments}

This study was financially supported by The $\mathrm{Na}$ tional Centre for Research and Development in terms of the project "Prevention Practises and Treatment of Civilizations Diseases-STRATEGMED" (grant no. STRATERMED2/269807/14/ NCBR/2015, acronim: BIOOPA.

\section{Conflict of interest}

The authors declare no conflict of interest.

\section{References}

1. Fine JD. Inherited epidermolysis bullosa. Orphanet J Rare Dis 2010; 5: 12.

2. Hernández-Martín A, Torrelo A. Inherited epidermolysis bullosa: from diagnosis to reality. Actas Dermosifiliogr 2010; 101: 495-505.

3. Fine JD, Bruckner-Tuderman L, Eady RA, et al. Inherited epidermolysis bullosa: updated recommendations on diagnosis and classification. J Am Acad Dermatol 2014; 70: 1103-26.
4. Has C, Liu L, Bolling MC, et al. Clinical practice guidelines for laboratory diagnosis of EB. Br J Dermatol 2020; 182: 574-92.

5. Wertheim-Tysarowska K, Sobczyńska-Tomaszewska A, Kowalewski C, et al. The COL7A1 mutation database. Hum Mutat 2012; 33: 327-31.

6. Ryan TD, Ware SM, Lucky AW, et al. Left ventricular noncompaction cardiomyopathy and aortopathy in a patient with recessive dystrophic epidermolysis bullosa. Circ Heart Fail 2012; 5: e81-2.

7. Villa CR, Ryan TD, Collins JJ, et al. Left ventricular non-compaction cardiomyopathy associated with epidermolysis bullosa simplex with muscular dystrophy and PLEC1 mutation. Neuromuscul Disord 2015; 25: 165-8.

8. Imbernón-Moya A, Maseda-Pedrero R, Feito M, de Lucas R. Dilated cardiomyopathy in a child with recessive dystrophic epidermolysis bullosa. Actas Dermosifiliogr 2019; 110: 81-3.

9. Cipriani A, Carrer A, De Lazzari M, et al. Arrhythmogenic cardiomyopathy in epidermolysis bullosa: are desmosomes at fault for both? Eur Heart I Cardiovasc Imaging 2019; 20 (Supplement 2).

10. Oh SW, Lee JS, Kim MY, et al. Recessive dystrophic epidermolysis bullosa associated with dilated cardiomyopathy. Br J Dermatol 2007; 157: 610-2.

11. Lara-Corrales I, Pope E. Dilated cardiomyopathy in epidermolysis bullosa. Dermatol Clin 2010; 28: 347-51.

12. Batalla A, Vicente A, Bartrons J, et al. Cardiomyopathy in patients with hereditary bullous epidermolysis. Actas Dermosifiliogr 2017; 108: 544-9.

13. Morelli S, Dianzani C, Sgreccia A, et al. Reversible acute global left ventricular dysfunction in a patient with autosomal recessive dystrophic epidermolysis bullosa. Int J Cardiol 2001; 79: 321-3.

14. Lara-Corrales I, Mellerio JE, Martinez AE, et al. Dilated cardiomyopathy in epidermolysis bullosa: a retrospective, multicenter study. Pediatr Dermatol 2010; 27: 238-43.

15. Grilletta EA. Cardiac transplant for epidermolysis bullosa simplex with KLHL24 mutation-associated cardiomyopathy. JAAD Case Rep 2019; 5: 912-4.

16. Melville C, Atherton D, Burch M, et al. Fatal cardiomyopathy in dystrophic epidermolysis bullosa. Br J Dermatol 1996; 135: 603-6.

17. Shipman AR, Moss C, Chickermane A, Browne F. Fatal dilated cardiomyopathy in a patient with recessive dystrophic epidermolysis bullosa. J Am Acad Dermatol 2015, 72 Suppl 1: AB106.

18. Lipiec P, Bąk J, Braksator W, et al. Transthoracic echocardiography in adults - guidelines of the working group on echocardiography of the Polish Cardiac Society. Kardiol Pol 2018; 76: 488-93.

19. Lang RM, Badano LP, Mor-Avi V, et al. Recommendations for cardiac chamber quantification by echocardiography in adults: an update from the American Society of Echocardiography and the European Association of Cardiovascular Imaging. Eur Heart J Cardiovasc Imaging 2015; 16: 233-71.

20. Nagueh SF, Smiseth OA, Appleton CP, et al. Recommendations for the evaluation of left ventricular diastolic function by echocardiography: an update from the American Society of Echocardiography and the European Association of Cardiovascular Imaging. J Am Soc Echocardiogr 2016; 29: 277-314.

21. Tei C, Ling LH, Hodge DO. New index of combined systolic and diastolic myocardial performance: a simple and reproducible measure of cardiac function - a study in normals and dilated cardiomyopathy. J Cardiol 1995; 26: 357-66. 
22. Duzenli MA, Ozdemir K, Aygul N, et al. Comparison of myocardial performance index obtained either by conventional echocardiography or tissue Doppler echocardiography in healthy subjects and patients with heart failure. Heart Vessels 2009; 24: 8-15.

23. Lopez L, Colan SD, Frommelt PC, et al. Recommendations for quantification methods during the performance of a pediatric echocardiogram: a report from the pediatric measurements Writing Group of the American Society of Echocardiography Pediatric and Congenital Heart Disease Council. J Am Soc Echocardiogr 2010; 23: 465-95.

24. Ryan TD, Lucky AW, King EC, et al. Ventricular dysfunction and aortic dilation in patients with recessive dystrophic epidermolysis bullosa. Br J Dermatol 2016; 174: 671-3.

25. Fine JD, Hall M, Weiner M, et al. The risk of cardiomyopathy in inherited epidermolysis bullosa. Br J Dermatol 2008; 159: 677-82.

26. Sharratt GP, Lacson AG, Cornel G, Virmanil S. Echocardiography of intracardiac filling defects in infants and children. Pediatr Cardiol 1986; 7: 189-94.

27. Sidwell RU, Yates R, Atherton D. Dilated cardiomyopathy in dystrophic epidermolysis bullosa. Arch Dis Child 2000; 83: 59-63.

28. Schwieger-Briel A, Fuentes I, Castiglia D, et al. Epidermolysis bullosa simplex with KLHL24 mutations is associated with dilated cardiomyopathy. J Invest Dermatol 2019; 139: 244-9.

29. Auckburally SH, Khan H, Martinez AE, et al. Should the frequency of echocardiogram screening be increased in severe subtypes of epidermolysis bullosa? Arch Dis Child 2017; 102 (Suppl 1): A1-218. 